\title{
Spectra of Euclidean Random Matrices
}

\author{
M. Mézard ${ }^{(1,2)}$, G. Parisi ${ }^{(3)}$, A. Zee(1) \\ (1) Institute for Theoretical Physics, \\ University of California Santa Barbara, CA 93106-4030 (USA) \\ (2) CNRS, Laboratoire de Physique Théorique de l'ENS, \\ 24 rue Lhomond, 75231 Paris (France) \\ (3) Dipartimento di Fisica and Sezione INFN, \\ Università di Roma "La Sapienza", Piazzale Aldo Moro 2, I-00185 Rome (Italy)
}

(August 14, 2018)

We study the spectrum of a random matrix, whose elements depend on the Euclidean distance between points randomly distributed in space. This problem is widely studied in the context of the Instantaneous Normal Modes of fluids and is particularly relevant at the glass transition. We introduce a systematic study of this problem through its representation by a field theory. In this way we can easily construct a high density expansion, which can be resummed producing an approximation to the spectrum similar to the Coherent Potential Approximation for disordered systems.

\section{INTRODUCTION}

The theory of random matrices has found applications in many branches of physics [ [ [ ] . The most developed theory concerns matrices where the matrix elements are either independent random variables, as in Gaussian ensembles, or are taken with a statistical distribution which is invariant under some symmetry group. However in many physical applications, from vibration spectra of glasses [2]3] to instantaneous normal modes in liquids [4], electron hopping in amorphous semiconductors [2] or combinatorial optimization problems [5], one needs to compute the spectrum and the eigenstate properties of some random matrices which are of a different type: The disorder is due to the random positions of $N$ points, and the matrix elements are given by a deterministic function of the distances between the points. We shall call these matrices Euclidean Random Matrices(ERM).

Specifically we want to study the following mathematical problems: Consider $N$ points in a volume $V$ of a $d$-dimensional Euclidean space. For a given sample, characterized by the positions $x_{i}$ of the $N$ points $(i \in\{1 \ldots N\})$, we want to study the properties of the $N \times N$ random matrices $M$ defined as

$$
M_{i j}=f\left(x_{i}-x_{j}\right)-u \delta_{i j} \sum_{k} f\left(x_{i}-x_{k}\right),
$$

where $u$ is a real parameter which enables us to interpolate between the two most interesting cases $u=0,1$. The case where $u=0$ is the simplest mathematical problem with Euclidean-correlated matrix elements, the case where $u=1$ is the natural problem which appears when studying for instance vibration modes of an amorphous solid, instantaneous normal modes of a liquid, or random master equations. The main difference is that when $u=1$ the matrix $M$ has fluctuating diagonal terms, tailored in such a way that $\sum_{j} M_{i j}=0$ : the vector with all components equal to one is an eigenvector with zero eigenvalue, which expresses global translation invariance.

To fully specify the problem one needs to characterize the probability distribution of the random points $x_{i}$, as well as the function $f(x)$. In this paper we shall concentrate on the case where the points are uniformly distributed in a cubic box of size $L=V^{1 / d}$, without correlations. In many applications one will have to generalize the problem to the case where the $x_{i}$ 's are correlated, including a short distance repulsion. This is a much more complicated problem which we shall not address here. As for the function $f(x)$, we shall assume that it depends only on the distance $|x|$ and that it decays fast enough at large argument. In particular we shall assume that the Fourier transform $\tilde{f}(k) \equiv \int d x e^{i k \cdot x} f(x)$ is a well defined function at all $k$.

The general problem of ERM theory is to understand the statistical properties of the eigenvalues and the corresponding eigenvectors of $M$ in the large $N$ limit (taken at fixed density $\rho=N / V$ ). This is certainly a very rich problem. In particular one can expect that the eigenvectors will exhibit in dimension $d \geq 3$ some localized and delocalized regimes separated by a mobility edge [6]. Here we wish to keep to the much simpler question of the computation of the spectrum of $M$. We shall develop a field theory for this problem, check it at high and low densities, and use a Hartree type method to approximate the spectrum in the $u=0$ case. 


\section{FIELD THEORY}

The spectrum can be computed from the resolvent:

$$
R(z)=\frac{1}{N} \overline{\operatorname{Tr} \frac{1}{z-M}},
$$

where the overline denotes the average over the positions $x_{i}$. It is possible to write down a field theory using a replica approach. We shall compute $\Xi_{N} \equiv \overline{\operatorname{det}(z-M)^{-n / 2}}$, and deduce from it the resolvent by using the replica limit $n \rightarrow 0$. It is easy to show that one can write $\Xi_{N}$ as a partition function over replicated fields $\phi_{i}^{a}$, where $i \in\{1 \ldots N\}, a \in\{1 \ldots n\}$ :

$$
\begin{aligned}
\Xi_{N}= & \int \prod_{i=1}^{N} \frac{d x_{i}}{V} \int \prod_{i=1}^{N} \prod_{a=1}^{n} d \phi_{i}^{a} \\
& \exp \left(-\frac{z}{2} \sum_{i, a}\left(\phi_{i}^{a}\right)^{2}+\frac{1}{2} \sum_{i, j, a} f\left(x_{i}-x_{j}\right) \phi_{i}^{a} \phi_{j}^{a}-\frac{u}{2} \sum_{i, a}\left(\phi_{i}^{a}\right)^{2} \sum_{j} f\left(x_{i}-x_{j}\right)\right) .
\end{aligned}
$$

Let us introduce the bosonic fields $\psi_{a}(x)=\sum_{i=1}^{N} \phi_{i}^{a} \delta\left(x-x_{i}\right)$ and $\chi(x)=\sum_{i, a}\left(\phi_{i}^{a}\right)^{2} \delta\left(x-x_{i}\right)$, together with their respective Lagrange multiplier fields $\hat{\psi}_{a}(x)$ and $\hat{\chi}(x)$. One can integrate out the $\phi$ variables, leading to the following field theory for $\Xi_{N}$ :

$$
\Xi_{N}=\int D\left[\psi_{a}, \hat{\psi}_{a}, \chi, \hat{\chi}\right] A^{N} \exp \left(S_{0}\right)
$$

where

$$
\begin{aligned}
S_{0} & =i \sum_{a} \int d x \hat{\psi}_{a}(x) \psi_{a}(x)+\frac{1}{2} \int d x \hat{\chi}(x) \chi(x)+\frac{1}{2} \sum_{a} \int d x d y \psi^{a}(x) f(x-y) \psi^{a}(y), \\
A & =\int \frac{d x}{V}\left(\frac{2 \pi}{z+\hat{\chi}(x)}\right)^{n / 2} \exp \left[-\frac{1}{2} u \int d y f(x-y) \chi(y)-\frac{1}{2(z+\hat{\chi}(x))} \sum_{a} \hat{\psi}^{a}(x)^{2}\right] .
\end{aligned}
$$

It is convenient to go to a grand canonical formulation of the disorder: we consider an ensemble of samples with varying number of points, and compute the grand canonical partition function $\mathcal{Z}(\alpha) \equiv \sum_{N=0}^{\infty} \Xi_{N} \alpha^{N} / N$ ! which is equal to:

$$
\mathcal{Z}=\int D\left[\psi_{a}, \hat{\psi}_{a}, \chi, \hat{\chi}\right] \exp \left(S_{0}+S_{1}\right) \quad ; \quad S_{1} \equiv \alpha A
$$

Notice that we can also integrate out the $\psi$ field thus replacing $S_{0}$ by $S_{0}^{\prime}$, where

$$
S_{0}^{\prime}=-\frac{n}{2} \operatorname{tr} \log f+\frac{1}{2} \int d x \hat{\chi}(x) \chi(x)+\frac{1}{2} \sum_{a} \int d x d y \hat{\psi}^{a}(x) f^{-1}(x-y) \hat{\psi}^{a}(y),
$$

where $\log f$ is the logarithm of $f$ considered as an integral operator and $f^{-1}$ is the integral operator which is the inverse of $f$.

The expression (6) is our basic field theory representation. We shall denote by brackets the expectation value of any observable with the action $S_{0}+S_{1}$. As usual with the replica method we have traded the disorder for an interacting replicated system. The basic properties of the field theory are related to the properties of the original problem in a straightforward way. The average number of particles is related to $\alpha$ through $N=\alpha\langle A\rangle$, so that one gets $\alpha=N$ in the $n \rightarrow 0$ limit. From the generalized partition function $\mathcal{Z}$, one can get the resolvent $R(z)$ through:

$$
R(z)=-\lim _{n \rightarrow 0} \frac{2}{n N} \frac{\partial \log \mathcal{Z}}{\partial z}
$$




\section{HIGH DENSITY EXPANSION}

Let us first show how this field theory can be used to derive a high density expansion. We shall rescale $z$ as $z=\rho \hat{z}$, and rescale the $\chi$ fields as: $\hat{\chi}(x)=\rho \hat{c}(x), \chi(x)=c(x) / \rho$. The interacting part of the action, $S_{1}$, can then be expanded as:

$S_{1} \simeq \rho \int d x \mu(x)-\frac{u}{2} \int d x d y \mu(x) f(x, y) c(y)+\frac{u^{2}}{8 \rho} \int d x d y c(x) h(x, y) c(y)-\frac{1}{2} \int d x \frac{\sum_{a} \hat{\psi}_{a}(x)^{2}}{\hat{z}+\hat{c}(x)}$,

where we have introduced:

$$
\mu(x) \equiv\left(\frac{2 \pi}{\rho(\hat{z}+\hat{c}(x))}\right)^{-n / 2} \quad ; \quad h(x, y) \equiv \int d r f(x, r) \mu(r) f(r, y) .
$$

Performing the quadratic $c$ functional integral, one finds (here and in the following we drop all the irrelevant $-\hat{z}$ independent- constants):

$$
\begin{aligned}
\mathcal{Z} & \propto \int D\left[\hat{\psi}_{a}, \hat{c}\right] \\
& \quad \exp \left[\rho \int d x \mu(x)-\frac{1}{2} \int d x d y \epsilon(x) h^{-1}(x, y) \epsilon(y)-\frac{1}{2} \sum_{a} \int d x d y \hat{\psi}^{a}(x) G^{-1}(x, y) \hat{\psi}^{a}(y)\right],
\end{aligned}
$$

where $G^{-1}(x, y)=\delta(x-y) /(\hat{z}+\hat{c}(x))-f^{-1}(x, y)$ is the propagator of the $\hat{\psi}$ field, and the field $\epsilon(x)$ is defined as:

$\epsilon(x)=\frac{\sqrt{\rho}}{u}\left(\hat{c}(x)-u \int d y f(x-y) \mu(y)\right)=\frac{\sqrt{\rho}}{u}\left(\hat{c}(x)-u \tilde{f}(0)-\frac{u n}{2} \int d y f(x-y) \log (\hat{z}+\hat{c}(y))\right)$

We then change variables from $\hat{c}$ to $\epsilon$, which involves a Jacobian which is easily computed to order $n$. The $\hat{\psi}$ integral is Gaussian, and to leading order in $\rho$, the $\epsilon$ field can be neglected in $G$, as well as in $h$. The only term in which $\epsilon$ is relevant turns out to be $\int d x \mu(x)$, which is simplified by an expansion to order $\epsilon^{2}$. Gathering the various contributions one gets

$$
\begin{aligned}
\log \mathcal{Z} \simeq & -\frac{n N}{2}\left(\log (\hat{z}+u \tilde{f}(0))-\frac{u^{2}}{2 \rho} \frac{\int(d k) \tilde{f}(k)^{2}}{(\hat{z}+u \tilde{f}(0))^{2}}+\frac{1}{\rho} \frac{f(0)}{\hat{z}+u \tilde{f}(0)}\right. \\
& \left.-\frac{1}{\rho} \int(d k) \log \left[\frac{\hat{z}+u \tilde{f}(0)-\tilde{f}(k)}{\hat{z}+u \tilde{f}(0)}\right]+O\left(\frac{1}{\rho^{2}}\right)\right),
\end{aligned}
$$

where the first two terms come from the expansion of $\int d x \mu(x)$, the third one is the Jacobian, and the fourth one is the contribution of the determinant from the $\hat{\psi}$ fluctuations.

This gives for the resolvent:

$$
\begin{aligned}
R(z)=\frac{1}{\rho} \frac{1}{\hat{z}+u \tilde{f}(0)} & +\frac{1}{\rho^{2}}\left(\int(d k) \frac{\tilde{f}(k)}{(\hat{z}+u \tilde{f}(0)-\tilde{f}(k))(\hat{z}+u \tilde{f}(0))}\right. \\
& \left.-\frac{u f(0)}{(\hat{z}+u \tilde{f}(0))^{2}}+\frac{u^{2} \int d r f(r)^{2}}{(\hat{z}+u \tilde{f}(0))^{3}}\right)+O\left(\frac{1}{\rho^{3}}\right) .
\end{aligned}
$$

This result is valid in the large $\rho$ limit, whenever the resolvent parameter $z$ is scaled as $z=\rho \hat{z}$. It can be checked directly. We consider the original form (2) of the resolvent and expand it in a $1 / z$ series. At order $1 / z^{p+1}$, the first two leading terms in $\rho$ are:

$$
\begin{aligned}
\rho^{p}(-u \tilde{f}(0))^{p}+p(1-u) f(0) \rho^{p-1}(-u \tilde{f}(0))^{p-1} & +\rho^{p-1} \sum_{p^{\prime}=2}^{p} C_{p}^{p^{\prime}} \int d k[-u \tilde{f}(0)]^{p-p^{\prime}}[\tilde{f}(k)]^{p^{\prime}} \\
& +\rho^{p-1} u^{2} C_{p}^{2}(-u \tilde{f}(0))^{p-2} \int d r f(r)^{2}
\end{aligned}
$$

where $C_{p}^{p^{\prime}}=\Gamma(p+1) /\left(\Gamma\left(p^{\prime}+1\right) \Gamma\left(p-p^{\prime}+1\right)\right)$. One can see that in the large $\rho$ limit the series simplifies if we first scale $z$ proportionally to $\rho$. Writing $z=\rho \hat{z}$, we can resum the leading terms in the series, which gives back the same resolvent as (14). 
One can study with this method the eigenvalue density for eigenvalues $|\lambda| \sim O(\rho)$, by taking $z=\lambda+i \eta$ and computing the imaginary part of the resolvent in the small $\eta$ limit. For $\rho \rightarrow \infty$ one would get the trivial result for the eigenvalue density $P(\lambda)=\delta(\lambda+\rho u \tilde{f}(0))$. Including the leading large $\rho$ correction which we have just computed, we find that $P(\lambda)$ develops, away from the peak at $\lambda \sim-\rho u \tilde{f}(0)$, a component of the form:

$$
P(\lambda) \sim \frac{1}{\rho} \int(d k) \delta(\lambda-\rho(\tilde{f}(k)-u \tilde{f}(0))) .
$$

The result (16) is the one that one could guess using the following simple argument: introducing $\phi_{j}=\exp \left(i k x_{j}\right)$, one gets:

$$
\sum_{j} M_{i j} \phi_{j}=\lambda_{i} \phi_{i}, \quad \lambda_{i}=\sum_{j} e^{-i k\left(x_{i}-x_{j}\right)} f\left(x_{i}-x_{j}\right)-u \sum_{m} f\left(x_{i}-x_{m}\right) .
$$

In the large density regime it is reasonable to use the central limit theorem to approximate the above sum, which would show that $\phi$ is near to an eigenvector, with eigenvalue $\rho(\tilde{f}(k)-u \tilde{f}(0))$. Notice that for this argument to hold, the discrete sum giving $\lambda_{i}$ in (17) must sample correctly the continuous integral. This will be the case only when the density $\rho$ is large enough that the phase $-i k\left(x_{i}-x_{j}\right)$ doesn't oscillate too much from one point $x_{j}$ to a neighbouring one. This imposes that the spatial frequency $|k|$ be small enough: $|k| \ll \rho^{1 / d}$. This same condition is present in the field theory derivation. We assume that $\tilde{f}(k)$ decreases at large $k$, and we call $k_{M}$ the typical range of $k$ below which $\tilde{f}(k)$ can be neglected. Let us consider the corrections of order $\rho^{-2}$ in eq. (14). It is clear that, provided $\hat{z}$ is away from $-u \tilde{f}(0)$, the ratio of the correction term to the leading one is of order $k_{M}^{d} \rho^{-1}$, and the condition that the correction be small is just identical to the previous one. The large density corrections near to the peak $\lambda \sim-\rho u \tilde{f}(0)$ cannot be studied with this method.

\section{LOW DENSITY EXPANSION}

The low density expansion is also easily performed from the field theoretic representation. Since the interaction $\alpha A$ is proportional to $\rho$ in the $n \rightarrow 0$ limit, we can expand in powers of $\alpha$ :

$$
\begin{aligned}
\mathcal{Z}= & \int D\left[\hat{\psi}_{a}, \chi, \hat{\chi}\right] e^{S_{0}^{\prime}} \\
& {\left[1+\alpha \int \frac{d x_{0}}{V}\left(\frac{2 \pi}{z+\hat{\chi}\left(x_{0}\right)}\right)^{n / 2} \exp \left(-\frac{u}{2} \int d y f\left(x_{0}-y\right) \chi(y)-\frac{1}{2\left(z+\hat{\chi}\left(x_{0}\right)\right)} \sum_{a} \hat{\psi}^{a}\left(x_{0}\right)^{2}\right)+\ldots\right] }
\end{aligned}
$$

At first order we can perform the $\chi$ integral, which fixes $\hat{\chi}(x)=u f\left(x-x_{0}\right)$ and we get:

$$
\mathcal{Z}=1+\rho\left(\frac{2 \pi}{z}\right)^{n / 2}\left(\operatorname{det} K_{1}\right)^{-n / 2}
$$

where $K_{1}$ is the operator: $K_{1}(x, y)=\delta(x-y)-\delta\left(x-x_{0}\right) \delta\left(y-x_{0}\right) f(0) /(z+u f(0))$. Using (8), one finds $R(z)=1 /(z-(1-u) f(0))$, which is obviously the leading result at very low densities, such that the points are isolated. At second order, the expansion (18) involves a double integral over points $x_{0}, x_{1}$. The $\chi$ integral fixes $\hat{\chi}(x)=u f\left(x-x_{0}\right)+u f\left(x-x_{1}\right)$. One needs to study the determinant of the operator $K_{2}$ defined as:

$$
K_{2}=\delta(x-y)-\frac{f(x-y)}{z+\hat{\chi}(x)}\left(\delta\left(x-x_{0}\right)+\delta\left(x-x_{1}\right)\right)\left(\delta\left(y-x_{0}\right)+\delta\left(y-x_{1}\right)\right) .
$$

This gives after a simple computation the order $\rho$ correction to the resolvent:

$\frac{\rho}{2} \int d r\left(\frac{1}{z-[(1-u) f(0)+(1-u) f(r)]}+\frac{1}{z-[(1-u) f(0)-(1+u) f(r)]}-\frac{2}{z-(1-u) f(0)}\right)$

This corrects the leading small $\rho$ formula by adding the contribution due to pairs of points, a distance $r$ apart, which are isolated from all other points. This expansion can be easily carried out to higher order, the order $\rho^{k}$ involving the computation of a $k d$ dimensional integral. 


\section{VARIATIONAL APPROXIMATION}

In order to try to elaborate a general approximation for the spectrum, which interpolates between the high and low density limits, we have used a standard Gaussian variational approximation in the field theory representation, which appears under various names in the literature, like the Random Phase Approximation. We have developed it only in the case $u=0$. The field theory then simplifies since the $\chi$ and $\hat{\chi}$ fields can be integrated out. Changing $\hat{\psi} \rightarrow i \hat{\psi}$, we obtain:

$$
\mathcal{Z}=\int D\left[\hat{\psi}^{a}\right] \exp \left(S_{u=0}\right)
$$

where

$$
S_{u=0}=-\frac{1}{2} \int d x d y \sum_{a} \hat{\psi}^{a}(x) f^{-1}(x, y) \hat{\psi}^{a}(y)+\rho z^{-n / 2} \int d x \exp \left(\frac{1}{2 z} \sum_{a} \hat{\psi}^{a}(x)^{2}\right) .
$$

We look for the best quadratic action

$$
S_{v}=-(1 / 2) \sum_{a b} \int d x d y G_{a b}^{-1}(x, y) \hat{\psi}^{a}(x) \hat{\psi}^{b}(y)
$$

which approximates the full interacting problem, using the fact that the variational free energy $F_{v}=\left\langle S_{u=0}\right\rangle_{v}-\log \mathcal{Z}_{v}$ should be minimized. (Here $\mathcal{Z}_{v}=\int D[\hat{\psi}] \exp \left(S_{v}\right)$, and the expectation values $\langle.\rangle_{v}$ are meant as Boltzmann like averages with the measure $\exp \left(S_{v}\right)$ ). The variational free energy is easily obtained:

$$
\frac{F_{v}}{V}=\frac{1}{2} \operatorname{Tr} f^{-1} G-\rho z^{-n / 2} \int d x_{0}\left(\frac{\operatorname{det}(K)}{\operatorname{det}(G)}\right)^{1 / 2}-\frac{1}{2} \operatorname{Tr} \log G,
$$

where the operator $K$ is defined as:

$$
K_{a b}^{-1}(x, y)=G_{a b}^{-1}(x, y)-\frac{1}{z} \delta_{a b} \delta\left(x-x_{0}\right) \delta\left(y-x_{0}\right) .
$$

In (25), both $G$ and $K$ are considered as operators in $x$ space and in replica space. Keeping to translation invariant variational actions, the result is easily expressed in terms of the Fourier transform $G_{a b}(k)$ of the function $G_{a b}(r)$. One finds that:

$$
\frac{\operatorname{det}(K)}{\operatorname{det}(G)}=\exp \left[\operatorname{Tr}_{n} \log \left(1-\frac{H}{z}\right)\right]
$$

where $H_{a b}=G_{a b}(r=0)=\int(d k) G_{a b}(k)$ is a $n \times n$ matrix, and the trace $T r_{n}$ is a trace in replica space. This gives for the variational free energy:

$$
\frac{F_{v}}{V}=\frac{1}{2} \int(d k) \frac{\operatorname{Tr}_{n} G(k)}{f(k)}-\mu \exp \left(-\frac{1}{2} \operatorname{Tr}_{n} \log (1-H / z)\right)-\frac{1}{2} \int(d k) T r_{n} \log G(k)
$$

We have found a solution of the stationarity equations, $\partial F_{v} / \partial G_{a b}(x)=0$, of the form $G_{a b}(x)=$ $\delta_{a b} G(x)$, where the Fourier transform $\tilde{G}$ satisfies the self consistency equation:

$$
\tilde{G}(k)=\frac{f(k)}{1-C f(k)} \quad, \quad C=\frac{\rho}{z-\int(d k) \tilde{G}(k)}
$$

In terms of this function, the variational free energy density per replica is:

$$
\frac{F_{v}}{n V}=\frac{1}{2} \int(d k) \frac{\tilde{G}(k)}{\tilde{f}(k)}+\frac{\rho}{2} \log \left(1-\frac{1}{z} \int(d k) \tilde{G}(k)\right)-\frac{1}{2} \int(d k) \log \tilde{G}(k)
$$

and the resolvent is obtained from:

$$
R(z)=\frac{1}{z}+\frac{2}{\rho} \frac{\partial}{\partial z} \frac{F_{v}}{n V}=\frac{C}{\rho}=\frac{1}{z-\int(d k) \tilde{G}(k)}
$$

Formulas (29, 30,31) provide a closed set of equations which allow us to compute the Gaussian variational approximation to the spectrum for any values of $f$ and the density. In sect. VI we shall 
compare this approximation to some numerical estimates of the spectrum for various functions $f$ and densities. It is easily checked that the variational gives the correct spectrum to leading order in the large $\rho$ expansion (see (14)) and in the small $\rho$ expansion, which is not a surprise since we had seen that these leading orders are given by purely Gaussian theories. Of course it fails to give the exact result beyond leading order, but it provides a reasonable interpolation between these extremes.

Another partial resummation of the $\rho$ expansion can also be done in the following way: if one neglects the triangular-like correlations between the distances of the points (an approximation which becomes correct in large dimensions, provided the function $f$ is rescaled properly with dimension), the problem maps onto that of a diluted random matrix with independent elements. This problem can be studied explicitly using the methods of [7] 12]. It leads to integral equations which can be solved numerically. The equations one gets correspond to the first order in the virial expansion in eq. (3), in which one introduces as variational parameter the local probability distribution of the field $\phi$. The detailed computations involving this other approximation scheme are left for future work.

\section{NUMERICAL SIMULATIONS}

For a function $f(x)$ which is positive, as well as its Fourier transform $\tilde{f}(k)$, simple bounds on the spectrum can be derived in the two cases $u=0$ and $u=1$. In the $u=0$ case, calling $\psi_{i}$ a normalised eigenvector $\psi_{i}$ of the matrix $M$ defined in (11), with eigenvalue $\lambda$, one has:

$$
\sum_{i j} \psi_{i} f\left(x_{i}-x_{j}\right) \psi_{j}=\lambda
$$

and the positivity of the Fourier transform of $f$ implies that $\lambda \geq 0$. In the $u=1$ case, the eigenvalue equation implies that:

$$
\sum_{j(\neq i)}\left|M_{i j}\right|\left|\psi_{j}\right| \geq\left|\lambda-M_{i i}\right|\left|\psi_{i}\right| .
$$

Summing over $i$, using the fact that $M_{i j}$ is positive for $i \neq j$, together with the constraint $\sum_{j} M_{i j}=$ 0, one gets:

$$
\sum_{j}\left(\left|M_{j j}\right|-\left|\lambda-M_{j j}\right|\right)\left|\psi_{j}\right| \geq 0
$$

which implies that there exists at least one index $j$ such that $\left|M_{j j}\right|-\left|\lambda-M_{j j}\right| \geq 0$, and therefore $\lambda \leq 0$. Furthermore, in this $u=1$ case, there exists one eigenvector (the uniform one) with zero eigenvalue.

In the $u=0$ case, we have studied numerically the problem in dimension $d=3$ with the Gaussian function $f(x)=(2 \pi)^{-3 / 2} \exp \left(-x^{2} / 2\right)$. In this Gaussian case the high density approximation gives a spectrum

$$
P(\lambda) \sim \frac{1}{\rho \pi^{2}} \frac{1}{\lambda}\left(\frac{1}{2} \log \frac{\rho}{\lambda}\right)^{1 / 2} \theta(\rho-\lambda)
$$

Notice that this spectrum is supposed to hold away from the small $\lambda$ peak, and in fact it is not normalizable at small $\lambda$.

The variational approximation computation described in the previous section is more involved. From (29,30,31), one needs to solve, given $z=\lambda-i \epsilon$, the following equations giving the complex function of $z, C(z)$ which we write as $C=a+i b$ :

$$
\begin{aligned}
& \lambda=\rho \frac{a}{a^{2}+b^{2}}+\frac{1}{2 \pi^{2}} \int_{0}^{\infty} k^{2} d k \frac{e^{k^{2} / 2}-u}{\left(e^{k^{2} / 2}-a\right)^{2}+b^{2}} \\
& \epsilon=\rho \frac{b}{a^{2}+b^{2}}-\frac{b}{2 \pi^{2}} \int_{0}^{\infty} k^{2} d k \frac{1}{\left(e^{k^{2} / 2}-a\right)^{2}+b^{2}} .
\end{aligned}
$$

One needs to find a solution in the limit where $\epsilon \rightarrow 0$. We have done this as follows: For a given value of $a$, we search for the values of $b$ which solve the second of eqs.(36) with $\epsilon=0$. Depending on 
the value of $a$, there may exist zero, one, or two solutions in $b$. For each such solution we compute the value of $\lambda$ from the first of qs.(36), and the corresponding density of states is given by $b /(\rho \pi)$.

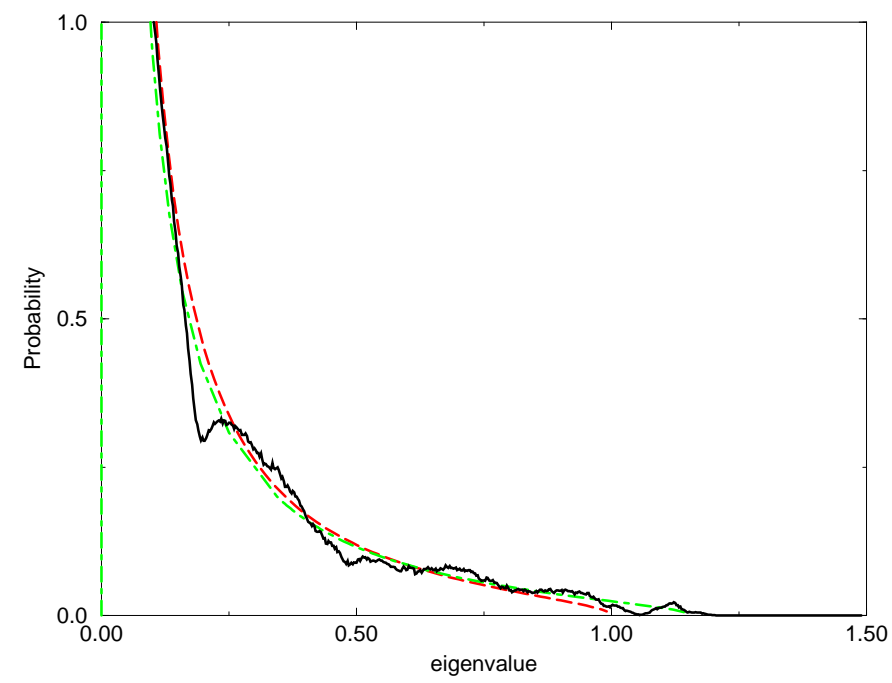

FIG. 1. Density of eigenvalues of a Euclidean Random Matrix in three dimensions, density $\rho=1$. The function $f$ is $f(x)=(2 \pi)^{-3 / 2} \exp \left(-x^{2} / 2\right)$, and the matrix is defined from eq.(西) with $u=0$. The full line is the result of a numerical simulation with $N=800$ points, averaged over 100 samples. The dashed (red) line is the result from the high density expansion. The dash-dotted (green) line is the result from the Gaussian variational approximation (RPA) to the field theory.

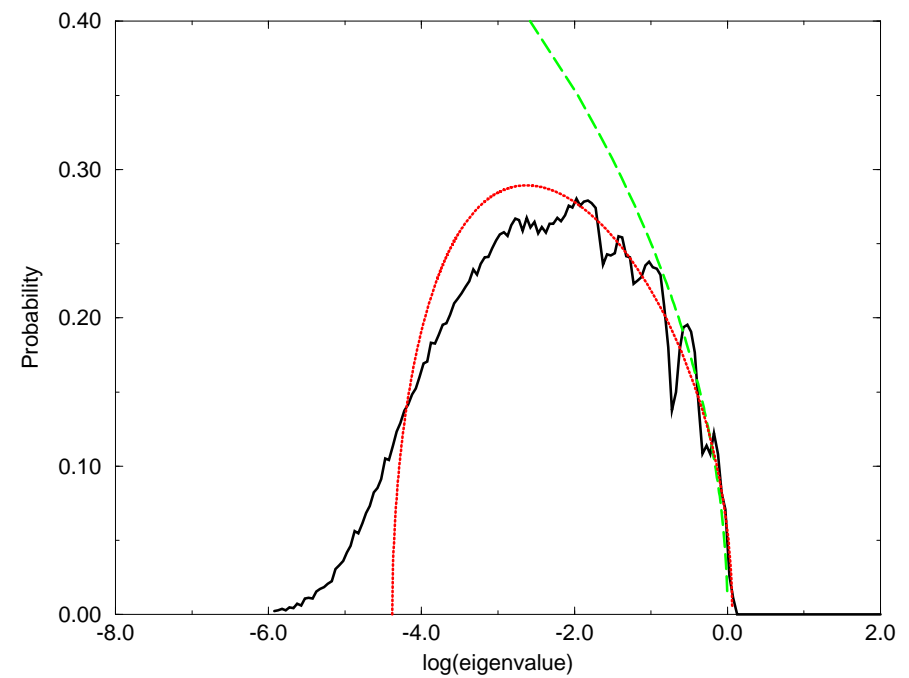

FIG. 2. Density of the logarithm (in base 10) of the eigenvalues of a Euclidean Random Matrix in three dimensions, density $\rho=1$. The function $f$ is $f(x)=(2 \pi)^{-3 / 2} \exp \left(-x^{2} / 2\right)$, and the matrix is defined from eq.(酉) with $u=0$. The full line is the result of a numerical simulation with $N=800$ points, averaged over 100 samples. The dashed (red) line is the result from the high density expansion. The dotted (green) line is the result from the Gaussian variational approximation (RPA) to the field theory.

In fig. (1.2), we plot the obtained spectrum, averaged over 100 realizations, for $N=800$ points at density $\rho=1$ (We checked that with $N=600$ points the spectrum is similar). Also shown 
are the high density approximation (35), and the result from the variational approximation. We see from fig.(11) that the part of the spectrum $\lambda \in[0.2,1.5]$ is rather well reproduced from both approximations, although the variational method does a better job at matching the upper edge. On the other hand the probability distribution of the logarithm of the eigenvalues (fig. (2) makes it clear that the high density approximation is not valid at small eigenvalues, while the variational approximation gives a sensible result. One drawback of the variational approximation, though, is that it always produces sharp bands with a square root singularity, in contrast to the tails which are seen numerically.

In fig. 3, we plot the obtained spectrum, averaged over 200 realizations, for $N=800$ points at density $\rho=0.1$ (We have checked that there is no substantial variation of the plot when going from $N=600$ to $N=800$ ). Also shown are the low density approximation (21), and the result from the variational approximation. We see from fig.(3) that this value of $\rho=0.1$ is more in the low density regime, and in particular there exists a peak around $\lambda=f(0)$ due to the isolated clusters containing small number of points. The variational approximation gives the main orders of magnitude of the distribution, but it is not able to reproduce the details of the spectrum, in particular the peak due to small clusters. On the other hand the leading low density approximation, which is not normalizable, gives a poor approximation at this intermediate density.

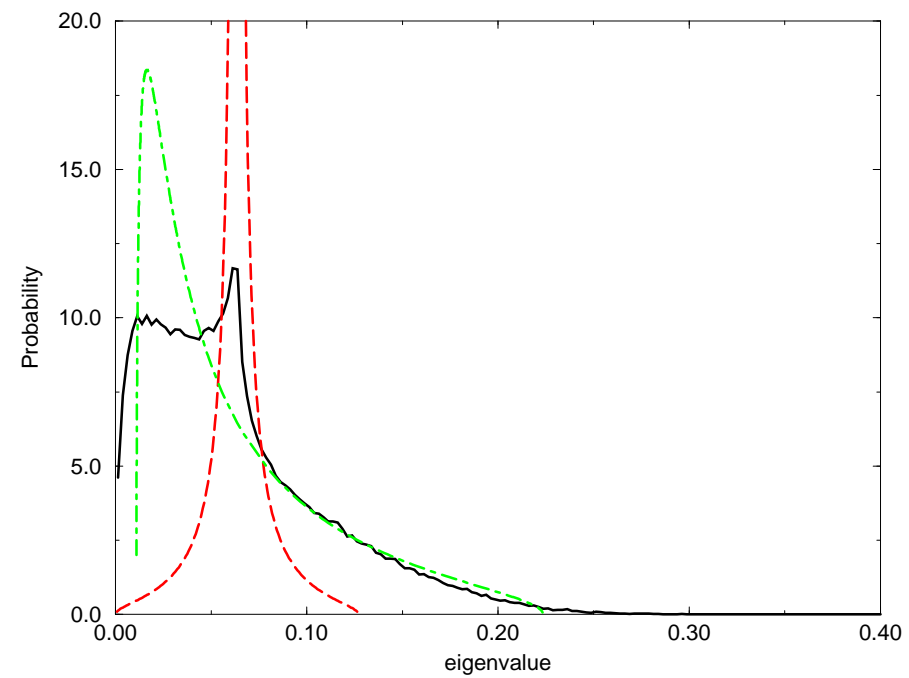

FIG. 3. Density of eigenvalues of a Euclidean Random Matrix in three dimensions, density $\rho=0.1$. The function $f$ is $f(x)=(2 \pi)^{-3 / 2} \exp \left(-x^{2} / 2\right)$, and the matrix is defined from eq.(1) with $u=0$. The full line is the result of a numerical simulation with $N=800$ points, averaged over 200 samples. The dashed (red) line is the result from the low density expansion. The dash-dotted (green) line is the result from the Gaussian variational approximation (RPA) to the field theory.

We now turn to the $u=1$ case. We have run some simulation in $d=3$ with the same Gaussian $f$ of width one, at density $\rho=1$. As seen in fig. 0 , the direct simulations with periodic boundary conditions, and sizes $N=1000$ to 1400 , show roughly the same density of states, with a very broad peak around $\lambda=-1=-\rho \tilde{f}(0)$. However they disagree in the tail of the spectrum near to the zero eigenvalue. In this region the finite size effects are more pronounced because of the gap opening at momenta less than $2 \pi(N / \rho)^{-1 / 3}$, which is rather large for the accessible values of $N$. In order to get a more precise result, less sensitive to the finite sizes, in this region of the spectrum, we have run some simulations which deal with an infinite set of points. Starting from a given sample of $N$ points in a box of side $L=(N / \rho)^{1 / 3}$, one imagines buiding up an infinite cubic lattice, for which the unit cell is the original box. Bloch's theorem tells that there exist $N$ bands, and the eigenstates of the infinite system are the products of a periodic function of period $L$ (in each direction) times a plane wave $e^{i p x_{j}}$. For a given value of the momentum $p \in[-\pi / L, \pi / L]^{3}$, the $N$ eigenvalues are those of the modified matrix:

$$
M_{i j}^{(p)}=f\left(d_{i j}\right) e^{i p \cdot \phi_{i j}}-u \delta_{i j} \sum_{k} f\left(d_{i k}\right)
$$


Here $d_{i j}$ is the distance between $x_{i}$ and $x_{j}$ with periodic boundary conditions, defined as the minimum over all translation vectors $t$ (translations of length multiple of $L$ in each direction) of $\left|x_{i}-x_{j}-t\right|$. The phase $\phi_{i j}$ is a d-dimensional vector. Its component in direction $\mu$ is related to the optimum translation $t$ through: $\phi_{i j}^{\mu}=t^{\mu} / L$. Given $p$, the problem is thus mapped to finding the spectrum of a $L^{d} \times L^{d}$ matrix $M^{(p)}$, with randomly twisted boundary conditions. The resulting matrix is hermitian and can be diagonalized by standard library routines. We have averaged the spectra over many samples, choosing for each sample one random Bloch momentum. This method 13. allows to access the small eigenvalue region of the spectra. As we see from fig. 4 , this whole region is well approximated by the high density approximation $(\sqrt{16})$, but this approximation fails to reproduce the broad peak of the spectrum, as expected.
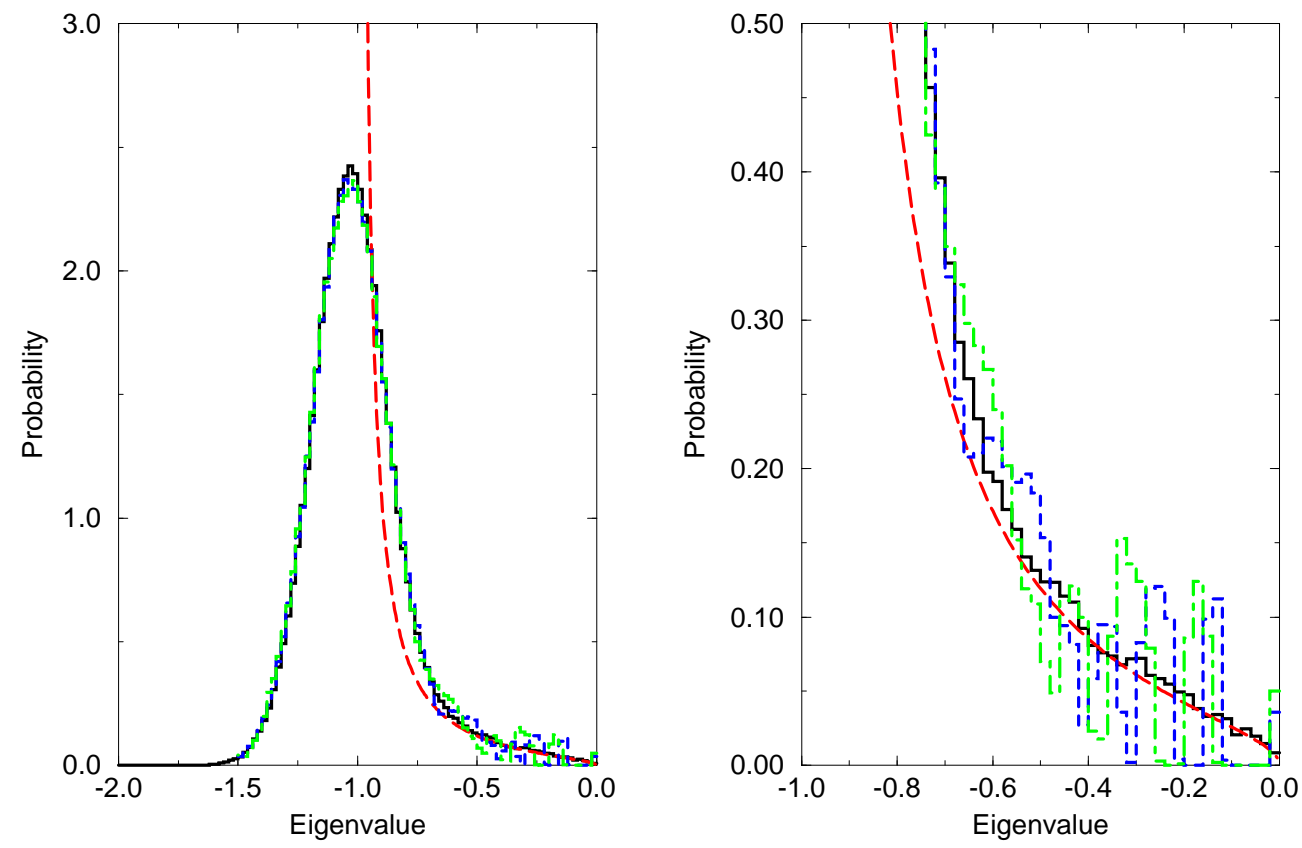

FIG. 4. Density of eigenvalues of a Euclidean Random Matrix in three dimensions, density $\rho=1$. The function $f$ is $f(x)=(2 \pi)^{-3 / 2} \exp \left(-x^{2} / 2\right)$, and the matrix is defined from eq.(位) with $u=1$. The right hand graph gives an enlargement of the spectrum at small eigenvalues. The full line is the result of a numerical simulation with quenched-twisted boundary conditions (see text), with $N=500$ points, averaged over 500 samples. The dashed (red) line is the result from the high density expansion. The dash-dotted (green) line is the result of a simulation with untwisted periodic boundary conditions, with $N=1000$ points averaged over 50 samples. The dotted (blue) line is the result of a similar simulation, but with $N=1400$ points.

To summarize, we have introduced a family of random matrices with Euclidean correlations, and developed for them a field theory representation, as well as some systematic expansions giving some properties of the eigenvalue density. The specra can be rather well approximated in the simplest case $u=0$ using some variational approximation to the field theory. In the most interesting case $u=1$, the situation is more complicated and we could get only the behaviour of the spectrum at small eigenvalues, using the high density expansion. It would certainly be interesting to generalize the variational approximation in order to treat also this $u=1$ case. 
[1] M.L. Metha, Random Matrices Academic Press, 1991.

[2] See e.g. S.R. Elliott, "Physics of amorphous materials", Longman (England, 1983), and references therein.

[3] M. Mézard and G.Parisi, Phys. Rev. Lett. 82 (1999)747.

[4] T.-M. Wu and R.F. Loring, J. Chem. Phys. 97 (1992) 8568; Y. Wan and R. Stratt, J. Chem Phys. $100(1994) 5123$.

[5] M. Mézard and G.Parisi, J. Physique 49 (1988) 2019.

[6] P.A. Lee and T.V. Ramakrishnan, Rev. Mod. Phys. 57 (1985) 287, and references therein.

[7] R. Abou-Chacra, P.W. Anderson and D.J. Thouless, J. Phys. C 6 (1973)1734.

[8] A.J. Bray and G.J. Rodgers, Phys. Rev. B38 (1988) 11461.

[9] Y.V. Fyodorov and A.D. Mirlin, Phys. Rev. Lett. 67 (1991) 2049.

[10] J.-P. Bouchaud and P. Cizeau, Phys.Rev. E50 (1994) 1810.

[11] G. Biroli and R. Monasson, cond-mat/9902032.

[12] A. Cavagna, I. Giardina and G. Parisi, cond-mat/9903155.

[13] G. Parisi, Phys. lett. 112B (1982) 463. 